\title{
Error Estimates to A Kind of Boundary Value Problem \\ about An Elliptic Differential Equation with Variable Coefficient
}

\author{
Rao Li, Yiming Chen, Yong Li \& Wei Guan \\ College of Science \\ Yanshan University \\ Qinhuangdao, 066004, China \\ E-mail: lirao123@yahoo.com.cn
}

\begin{abstract}
A kind of boundary value problem about a second-order elliptic differential equation with variable coefficient is discussed indirectly by transforming it as the second kind of variational inequality form. Using regularization method, the variational inequality is formulated as an equal variational equation, which can be made discrete by the finite element method. Abstract error estimate and the error estimates of the approximation are derived under the energy norm and $L^{2}$-norm.
\end{abstract}

Keywords: Variational inequality, Error estimate, Finite element analysis, Elliptic differential equation

\section{Problem Statement}

Suppose $\Omega \in R^{n}$ is a bounded open domain, $\Gamma$ is the sufficiently smooth boundary of $\Omega$, and $n$ is the outward normal to $\Gamma \quad, \quad \operatorname{meas}(\Gamma)>0 ; \quad V=\left\{u \in H^{1}(\Omega), u \geq 0\right\} \quad ; \quad K \subset V \quad$; $\quad$ Where $a(u, v)=\int_{\Omega} \sum_{i, j=1}^{n} \alpha_{i j}(x) \frac{\partial u}{\partial x_{j}} \frac{\partial v}{\partial x_{i}} d x+\int_{\Omega} \beta(x) u v d x, j(v)=g \sum_{i, j=1}^{n} \alpha_{i j}(x)|v| d s, g$ is a positive constant; $\langle f, v\rangle=\int_{\Omega} f v d x, f \in L^{n}(\Omega)$. In this paper, we discuss the boundary value problem about a second-order elliptic type differential equation with variable coefficient as follows:

$$
\begin{cases}-\sum_{i, j=1}^{n} \frac{\partial}{\partial x_{i}}\left[\alpha_{i, j}(x) \frac{\partial u(x)}{\partial x_{j}}\right]+\beta(x) u(x)=f(x) \quad \text { in } \Omega \\ \left|\frac{\partial u}{\partial n_{A}}\right| \leq g & \text { on } \Gamma \\ u \frac{\partial u}{\partial n_{A}}+g|u|=0 & \text { on } \Gamma\end{cases}
$$

The coefficients and the solution $u$ in (1) satisfy:

(i) Exist $a>0$, such that $\sum_{i, j=1}^{n} \alpha_{i j}(x) \xi_{i} \xi_{j} \geq a \sum_{i=1}^{n} \xi_{i}^{2}$,a.e. $x \in \Omega, \forall \xi \in R^{n}$.

(ii) $\alpha_{i j}(x) \in L^{\infty}(\Omega), \alpha_{i j}(x)=\alpha_{j i}(x), \beta(x) \in L^{\infty}(\Omega), \frac{\partial u}{\partial x_{j}} n_{j}=\frac{\partial u}{\partial n_{A}} \in H^{-\frac{1}{2}}(\Gamma)$.

We had proven the variational inequality problem that is equal to problem (1), as follows:

$\left\{\begin{array}{l}\text { find } u \in V \text {, such that } \\ a(u, v-u)+j(v)-j(u) \geq\langle f, v-u\rangle \forall v \in V\end{array}\right.$

And the variational inequality exists only one solution. (Chen, 2008)

Problem (2) originates from many physics and engineering reality. The variational inequality includes an 
indifferentiable functional. Using the regularization method (Chen, 2008), and making use of the differentiable function (R. Ding, 2005, pp.121-124), this problem was formulated as an equal variational equation. We will construct approach by finite element method and give the abstract error estimate and the error estimates of the approximation under the energy norm and $L^{2}$-norm. For better dissertating, regularization about the variational inequality (2) and the process of solving the equal variational equation (Chen, 2008) will be iterated in the next part briefly.

\section{Regularization method and the equal problem}

Using regularization method, we construct the differentiable functional $j_{\mathcal{E}}(v)=g \sum_{i, j=1}^{n} \int_{\Gamma} \alpha_{i j}(x) \psi_{\mathcal{\varepsilon}}(v) d s$ to substitute $j(v)=g \sum_{i, j=1}^{n} \alpha_{i j}(x)|v| d s$ approximately, where

$\psi_{\varepsilon}(\xi)=\int_{0}^{\xi} \varphi(t) d t=\left\{\begin{array}{l}g \xi-\frac{1}{2} \varepsilon g^{2}, \xi \geq \varepsilon g \\ \frac{\xi^{2}}{2 \varepsilon},|\xi| \leq \varepsilon g \\ -g \xi-\frac{1}{2 \varepsilon} g^{2}, \xi \leq-\varepsilon g\end{array}\right.$

And

$$
\varphi(t)=\left\{\begin{array}{l}
g, \xi \geq \varepsilon g \\
\frac{t}{\varepsilon},|\xi| \leq \varepsilon g \\
-g, \xi \leq-\varepsilon g
\end{array}\right.
$$

Easily we know that $\lim _{\mathcal{E} \rightarrow 0} \psi_{\mathcal{E}}(v)=g|v|$. Problem (2) can be substituted approximately by the problem:

$\left\{\begin{array}{l}\text { find } u \in V, \text { such that } \\ a(u, v-u)+j_{\varepsilon}(v)-j_{\varepsilon}(u) \geq\langle f, v-u\rangle \forall v \in V\end{array}\right.$

We can easily prove that $j_{\mathcal{\varepsilon}}(v)$ is convex, differentiable and $\lim _{\varepsilon \rightarrow 0} j_{\mathcal{E}}(v)=j(v)$.

The following theorem is quoted from the article (Chen, 2008), we give it directly without proof.

Theorem 1 Suppose $u$ and $u_{\varepsilon}$ are the solutions of problem (2) and (5) respectively, then when $\varepsilon \rightarrow 0, u_{\varepsilon}$ converqs to $u$ strongly.

In the following we consider the equal form to variational inequality (5).

Let $v=u \pm t w, t>0, w \in V$ in (5), then we have

$$
\pm a(u, w)+g \sum_{i, j=1}^{n} \int_{\Gamma} \alpha_{i j}(x)\left[\frac{\psi_{\varepsilon}(u \pm t w)-\psi_{\varepsilon}(u)}{t}\right] d s \geq \pm\langle f, v\rangle
$$

Let $t \rightarrow 0^{+}$, we have

$$
\lim _{t \rightarrow 0^{+}} \frac{\psi_{\varepsilon}(u \pm t w)-\psi_{\varepsilon}(u)}{t}= \pm \psi_{\varepsilon}^{\prime}(u) w= \pm \varphi(u) w .
$$

Then the solution $u \in V$ of problem (5) satisfies

$$
a(u, v)+g \sum_{i, j=1}^{n} \int_{-} \alpha_{i j}(x) \varphi(u) v d s=\langle f, v\rangle \quad \forall v \in V
$$

So we obtain the equal variational form (6) to problem (5).

\section{The finite element approach and the error estimate}

In order to carry on the stability estimate and the error analysis, we give the following lemma and the deduction at first. $C$ is used to express constant in the paper, and the identical letter appears in different place may express different constants.

Lemma 1 Function $\varphi(t)$ is defined as (4), then $\varphi(t) \in H^{1}(-\infty,+\infty)$ and satisfies Lipschitz and monotony conditions as 
follow:

$$
\begin{array}{ll}
|\varphi(u)-\varphi(v)| \leq \frac{1}{\varepsilon}|u-v| & \forall u, v \in R \\
(\varphi(u)-\varphi(v))(u-v) \geq 0 & \forall u, v \in R
\end{array}
$$

Deduction 1 The solution of problem (6) exists uniquely and satisfies the following stability:

$\|u\|_{1} \leq C\|f\|$

Proof The solution of problem (5) exists uniquely. Form the equivalence between (5) and (6), we know that problem (6) has only one solution. Following we prove the stability estimate. Take $v=u$ in (6), and take notice of $\varphi(0)=0$ form (9), by lemma 1, we have $\varphi(u) u \geq 0$, also by $g>0, \alpha_{i j} \geq 0$ a.e.on $\Gamma$, we can obtain $a(u, u) \leq\langle f, u\rangle$. Additionally, as the bilinear form $a(u, u)$ is compulsive, we can obtain the stability estimate.

Suppose $J_{h}$ is a regular triangulation in $\Omega, V_{h} \subset V$ is the corresponding linear finite element space. Marking $\|u\|_{E}=\sqrt{a(u, u)}$ to express the energy norm. Consider the following finite element approximation problem about problem (6).

$$
\left\{\begin{array}{l}
\text { find } u_{h} \in V_{h}, \text { such that } \\
a\left(u_{h}, v_{h}\right)+g \sum_{i, j=1}^{n} \int \alpha_{i j}(x) \varphi\left(u_{h}\right) v_{h} d s=\left\langle f, v_{h}\right\rangle \quad \forall v_{h} \in V_{h}
\end{array}\right.
$$

Just like deduction 1, we can obtain the existence and uniqueness of the solution of (10), and the solution similarly satisfies the stability estimate (9). Abstract error estimate about solutions of (6) and (10) will be given in the next part.

Theorem 2 Suppose $u$ and $u_{h}$ are the solutions of problem (6) and (10) respectively, then we have error estimates under the energy norm and $L^{2}$-norm as follows:

$$
\begin{aligned}
& \left\|u-u_{h}\right\|_{E} \leq C \inf _{v_{h} \in V_{h}}\left\{\left\|u-v_{h}\right\|_{E}+g \sum_{i, j=1}^{n}\left\|\alpha_{i j}(x)\right\|_{1}\left\|u-v_{h}\right\|_{L^{2}(\Gamma)}\right\} \\
& \left\|u-u_{h}\right\|_{L^{2}(\Omega)} \leq C \sum_{i, j=1}^{n}\left\|\alpha_{i j}(x)\right\|_{1} \sup _{q \in L^{2}(\Omega)}\left\{\frac{1}{\|q\|} \inf _{v_{h} \in V_{h}}\left\|\omega-v_{h}\right\|_{E}\right\}\left\|u-u_{h}\right\|_{E}
\end{aligned}
$$

For assigned $q \in L^{2}(\Omega), \omega \in V$ is the unique solution of the following elliptic problem (17).

Proof To perform subtraction between (6) and (10), we can obtain

$$
a\left(u-u_{h}, v_{h}\right)+g \sum_{i, j=1}^{n} \int_{\Gamma} \alpha_{i j}(x)\left[\varphi(u)-\varphi\left(u_{h}\right)\right] v_{h} d s=0 \quad \forall v_{h} \in V_{h}
$$

From this expression, using the compulsive character of the bilinear form $a(u, u)$, we have

$$
\begin{aligned}
\mu_{0}\left\|u-u_{h}\right\|_{E}^{2} \leq a\left(u-u_{h}, u-u_{h}\right) \\
=a\left(u-u_{h}, u-v_{h}\right)-g \sum_{i, j=1}^{n} \int_{\Gamma} \alpha_{i j}(x)\left[\varphi(u)-\varphi\left(u_{h}\right)\right]\left(v_{h}-u_{h}\right) d s \\
=a\left(u-u_{h}, u-v_{h}\right)-g \sum_{i, j=1}^{n} \int_{\Gamma} \alpha_{i j}(x)\left[\varphi(u)-\varphi\left(u_{h}\right)\right]\left(v_{h}-u\right) d s \\
-g \sum_{i, j=1}^{n} \int_{\Gamma} \alpha_{i j}(x)\left[\varphi(u)-\varphi\left(u_{h}\right)\right]\left(u-u_{h}\right) d s
\end{aligned}
$$

By Lemma1, the bounded character of $a(u, u)$ and trace theorem, we can obtain

$$
\begin{aligned}
\mu_{0}\left\|u-u_{h}\right\|_{E}^{2} & \left.\leq \mu_{1}\left\|u-u_{h}\right\|_{E}\left\|u-v_{h}\right\|_{E}+\frac{1}{\varepsilon} g \sum_{i, j=1}^{n} \int_{L} \alpha_{i j}(x)\right) u-u_{h} \| v_{h}-u \mid d s \\
& \leq \mu_{1}\left\|u-u_{h}\right\|_{E}\left\|u-v_{h}\right\|_{E}+C \frac{1}{\varepsilon} g \sum_{i, j=1}^{n}\left\|\alpha_{i j}(x)\right\|_{1}\left\|u-u_{h}\right\|_{E}\left\|v_{h}-u\right\|_{L^{2}(\Gamma)} \quad v_{h} \in V_{h}
\end{aligned}
$$


Thus we obtain the error estimate expression (11).

In order to derive the error estimate under $L^{2}$ norm, for discretionally assigned $q \in L^{2}(\Omega)$, we first introduce the following auxiliary problem

$$
\left\{\begin{array}{l}
-\sum_{i, j=1}^{n} \frac{\partial}{\partial x_{i}}\left[\alpha_{i, j}(x) \frac{\partial \omega(x)}{\partial x_{j}}\right]+\beta(x) \omega(x)=q \quad \text { in } \Omega \\
\frac{\partial \omega}{\partial n_{A}}+\gamma(x) \omega=0 \quad \text { оп } \Gamma
\end{array}\right.
$$

Where $\gamma(x)=\frac{\varphi(u)-\varphi\left(u_{h}\right)}{u-u_{h}}$. The equal variational form of problem (16) is

$$
\left\{\begin{array}{l}
\text { find } \omega \in V, \text { such that } \\
A(\omega, v)=\langle q, v\rangle \quad \forall v \in V
\end{array}\right.
$$

Where, $A(\omega, v)=a(\omega, v)+\sum_{i, j=1}^{n} \int \gamma \alpha_{i j}(x) \omega v d s$. We may know by lemma 1 that $\gamma(x) \in L^{\infty}(\Omega)$ and satisfies

$$
0 \leq \gamma(x) \leq \frac{1}{\varepsilon} \quad x \in \bar{\Omega}
$$

By $A(\omega, v)$, expression (18) and trace theorem, we know that the bilinear form $A(\cdot, \cdot)$ satisfies compulsive and bounded conditions in $V$, so the solution of problem (17) exists unequally.

Let $v=e=u-u_{h}$ in (17), using expression (13), the definition of $\gamma(x)$, lemma 1 and trace theorem, for $\forall v_{h} \in V_{h}$, we can obtain

$$
\begin{aligned}
(e, q) & =a(e, \omega)+g \sum_{i, j=1}^{n} \int_{\Gamma} \gamma \alpha_{i j}(x) \omega e d s=a\left(e, \omega-v_{h}\right)+a\left(e, v_{h}\right)+g \sum_{i, j=1}^{n} \int_{\Gamma} \gamma \alpha_{i j}(x) \omega e d s \\
& =a\left(e, \omega-v_{h}\right)-g \sum_{i, j=1}^{n} \int_{\Gamma} \alpha_{i j}(x)\left[\varphi(u)-\varphi\left(u_{h}\right)\right] v_{h} d s+g \sum_{i, j=1}^{n} \int_{T} \gamma \alpha_{i j}(x) \omega e d s \\
& =a\left(e, \omega-v_{h}\right)+g \sum_{i, j=1}^{n} \int_{\Gamma} \alpha_{i j}(x)\left[\varphi(u)-\varphi\left(u_{h}\right)\right]\left(\omega-v_{h}\right) d s \\
& \leq \mu\|e\|_{E}\left\|\omega-v_{h}\right\|_{E}+C \frac{1}{\varepsilon} g \sum_{i, j=1}^{n}\left\|\alpha_{i j}(x)\right\|_{1}\left\|u-u_{h}\right\|_{E}\left\|\omega-v_{h}\right\|_{E}
\end{aligned}
$$

By the haphazardness of $q \in L^{2}(\Omega)$ and $v_{h} \in V_{h}$, we have expression (12). The proof finished.

By problem (20) and the compulsory condition of $A(\omega, v)$, we can obtain the following lemma:

Lemma 2 When $\Omega$ is a convex region or a full smooth bounded domain, the solution of problem (17) $\omega \in V \cap H^{2}(\Omega)$ and satisfies the stability estimate $\|\omega\|_{2} \leq C\|q\|$.

In order to obtain the error estimate, we define interpolation operator $\Pi_{h}: C^{0}(\bar{\Omega}) \rightarrow V_{h}$ as follow: for $\forall v \in C^{0}(\bar{\Omega})$, $\Pi_{h} v \in V_{h}$ such that $\Pi_{h} v(P)=v(P), \forall$ dissection node $P \in \bar{\Omega}$.

By the above conclusions, theorem 2, lemmas 2 as well as the finite element interpolation theory, we can obtain conclusions about the error estimate as follows.

Theorem 3 Suppose $u \in V$ is the solution of problem (7) and $u_{h} \in V_{h}$ is the solution of (10), then when $u \in H^{1+\alpha}(\Omega), \alpha \geq 0$, the following error estimates come into existence:

$\left\|u-u_{h}\right\|_{E} \leq C h^{\alpha}\left(\|u\|_{1+\alpha}+g h^{\frac{1}{2}} \sum_{i, j=1}^{n}\left\|\alpha_{i j}(x)\right\|_{1}\|u\|_{H^{\frac{1}{2}+\alpha}(\Gamma)}\right)$ 


$$
\left\|u-u_{h}\right\|_{L^{2}(\Omega)} \leq C h^{1+\alpha} \sum_{i, j=1}^{n}\left\|\alpha_{i j}(x)\right\|_{1}\left(\|u\|_{1+\alpha}+g h^{\frac{1}{2}} \sum_{i, j=1}^{n}\left\|\alpha_{i j}(x)\right\|_{1}\|u\|_{H^{\frac{1}{2}+\alpha}(\Gamma)}\right)
$$

Proof Let $v_{h}=\Pi_{h} u$ in the abstract error estimate expression (11), then

$$
\begin{gathered}
\left\|u-u_{h}\right\|_{E} \leq C \inf _{v_{h} \in V_{h}}\left\{\left\|u-v_{h}\right\|_{E}+g \sum_{i, j=1}^{n}\left\|\alpha_{i j}(x)\right\|_{1}\left\|u-v_{h}\right\|_{L^{2}(\Gamma)}\right\} \\
\leq C\left\{\left\|u-\Pi_{h} u\right\|_{E}+g \sum_{i, j=1}^{n}\left\|\alpha_{i j}(x)\right\|_{1}\left\|u-\Pi_{h} u\right\|_{L^{2}(\Gamma)}\right\}
\end{gathered}
$$

By the finite element interpolation theory ${ }^{[3]}$, for $u \in H^{1+\alpha}(\Omega), \alpha \geq 0$, we have

$$
\left\|u-\Pi_{h} u\right\|_{E} \leq C_{0} h^{\alpha}\|u\|_{1+\alpha} \quad,\left\|u-\Pi_{h} u\right\|_{L^{2}(\Gamma)} \leq C_{1} h^{\frac{1}{2}+\alpha}\|u\|_{H^{\frac{1}{2}+\alpha}(\Gamma)}
$$

Substitute them into the right margin of (21), and then we have the error estimate (19).

Let $v_{h}=\Pi_{h} \omega$ in (12) and using lemma 2, we have

$$
\begin{aligned}
\left\|u-u_{h}\right\|_{L^{2}(\Omega)} & \leq C \sup _{q \in L^{2}(\Omega)}\left\{\frac{1}{\|q\|} \inf _{v_{h} \in V_{h}}\left\|\omega-v_{h}\right\|_{E}\right\} \sum_{i, j=1}^{n}\left\|\alpha_{i j}(x)\right\|_{1}\left\|u-u_{h}\right\|_{1} \\
& \leq C h \sup _{q \in L^{2}(\Omega)}\left\{\frac{1}{\|q\|}\|\omega\|_{2}\right\} \sum_{i, j=1}^{n}\left\|\alpha_{i j}(x)\right\|_{1}\left\|u-u_{h}\right\|_{E} \leq C h \sum_{i, j=1}^{n}\left\|\alpha_{i j}(x)\right\|_{1}\left\|u-u_{h}\right\|_{E}
\end{aligned}
$$

Additionally by (19) which have been proved above, we can obtain expression (20). The proof finished.

\section{Conclusions}

The variational equation equal to the variational inequality is made discrete by finite element method, which made the interpolation computation directly to the original variational inequality simplified. The existence, uniqueness and stability of the finite element solution are given. The abstract error estimate is established, and error estimate under the energy norm and $L^{2}$ norm is given. The finite element analysis about the variational inequality problem narrated in the paper is consummated.

\section{References}

Chen Y. M. and Li R.. "The Existence and Uniqueness of the Solution for a kind of Second Order Elliptic Differential Equation of Variable Coefficient". Third international conference on innovative computing, information and control. China. 2008.

Ding R. and Qian F. B.. "Regularization and finite element approximation for fourth-order variational inequality of the second kind." Journal of Lanzhou University (Natural Sciences). 2005. pp. 121-124.

Ciarlet P. G.. The finite element method for elliptic problems. North-Holland. Amsterdam. 1978.

Glowinski R, Lions J. L. and Tremolieres R. Numerical analysis of variational inequality. Amsterdam. North-Holland Publishing Company. 1981.

\section{Acknowledgement}

This work is supported by the Nature Science Foundation of Hebei province in China (E2007000381). 